$\xi=-1$

\title{
Multitudinous of remedial medical image using stationary wavelet transform
}

\author{
Mrs. S. Meera ${ }^{1 *}$, Mrs. R. Sharmikha Sree ${ }^{1}$, Mrs. R. Deepika ${ }^{1}$, Mrs. R. A. Kalpana ${ }^{1}$ \\ ${ }^{1}$ Assistant Professor, Dept. of CSE, Sri Sairam Engineering College \\ *Corresponding author E-mail: meera.cse@sairam.edu.in
}

\begin{abstract}
Image fusion is becoming more popular in various field nowadays. The quality of accuracy and perception has been achieved with this con-cept. The field that is in urgent need of more contrast images and quality output of body organs image reproduction is medicine. This paper proposes the concept of introducing the image fusion in the area of body imaging to get a more accurate and contrast images for the identifi-cation of the tumor or any other mal-functionalities in the human body. The image fusion is enhanced with the choice of more efficient and contrast uplifting technique called stationary wavelet form. This technique accepts the input of two different perceptive images of the finicky region and enhances their details in the resulting image. The advantageous part of the technique outputs the more detailed edge separation feature which further more allows the fast diagnosis of the any defect prevailing currently in the place subjected to the imaging test. This technique also paves the way to get the more reliable and meticulous result in the future when the world starts embracing the advantage of automation in the field of medicine.
\end{abstract}

Keywords: Image Fusion; Stationary Wavelet Transform; Efficient; DWT; Medical Field.

\section{Introduction}

In the recent years, image fusion has found its strong foundation in various fields and is in the way to strike its bell in the medical field. The vision can be broadly classified into two different types. And those two types are human vision and computer vision. The human vision more specifically acts via the stimuli and is more sophisticated system of vision and senses. Moreover it is more based on the neurons and other nerves. Whereas a computer vision highly needs a computer, an interface and a computer.

The feature that more basically separates the two visions is the measure of efficiency and quality of the perceived output. It always tends to be such that the computer vision has another added advantage of recording the image in any of the formats that can anytime be retrieved later for the future use.

The process of recording the image and using it for future use has always been of very vital use in various fields. And the static way of representing the image from a single perspective or angle, denied the particulars that happened to be the actual objective of the need of hour.

Later, the idea of combining the various images of the same distinct object, captured from different angles yielded various breathtakingly contrast images as the result. Thus evolved the idea of image fusion. The key factor of fusing two images availed to be of the same subject, captured from various productive angles, that happens to throw light on the more detailed version of the expected image. This method of fusion of images can be attained with various techniques. The most basic operations that can be performed while fusing two images are: 1.Addition, 2.Average, 3.Subtraction, 4.Multiplication.

The evolution of the image fusion can be shown as follows:

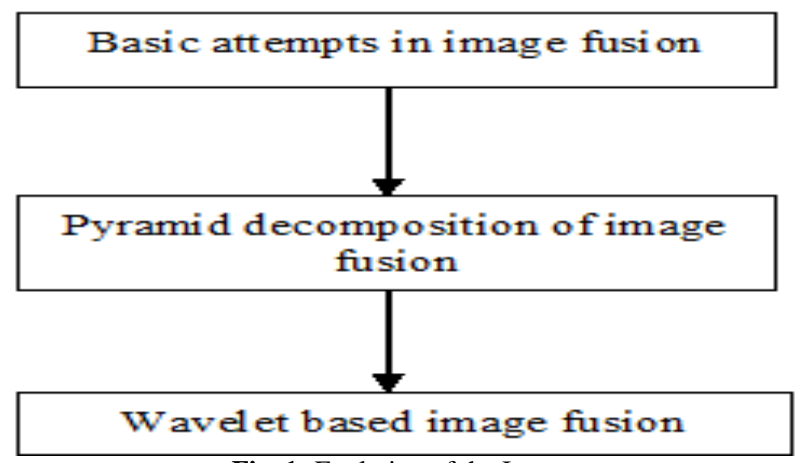

Fig. 1: Evolution of the Image.

The pyramid decomposition based image fusion was implemented in the mid 80's. It brought over a bright light on the various beauties of the fused image. The more detailed version was obtained as a result but the major drawback that was witnessed was the reduction in the contrast of the resulting image which was very disappointing rather. Then various researches were made that ended up with the mathematical transforms under usage for the fusion.

It involved the transform equations to be applied on the input images and separate them into various levels of components such that the information are grouped together in various portions of the segregated components. The resulting image seemed to be more informative about the edges of the object.

\section{Basic idea}

To obtain the scanning output of the organ without any error while displaying, the MRI (Magnetic Resonance Imaging) is used to provide the contrast color distortion of the image and the better information on the soft tissues. It is very useful and advanced 
while taking the fusion process's input into consideration. And also the content for the fusion is exactly provided by this type of scan. CT (Computed Tomography) will produce cross sectional images of some particular area of the scanned image object and also measures the different angle of the same subject ad provides the better information about the denser tissue with less color distortion.

PET (Positron Emission Tomography) is another of the scanning techniques that helps in the figuring of metabolic changes and pseudo color. Both will be separated as two images to identify the difference and exact area of he disease location. MR scan which is used to verify the location of the disease affected area and PET image is used to analyze and observe the metabolic changes in the medical images by combining and integrating the process of both PET and MRI into single image can reduce the work of the long process to identify the disease.

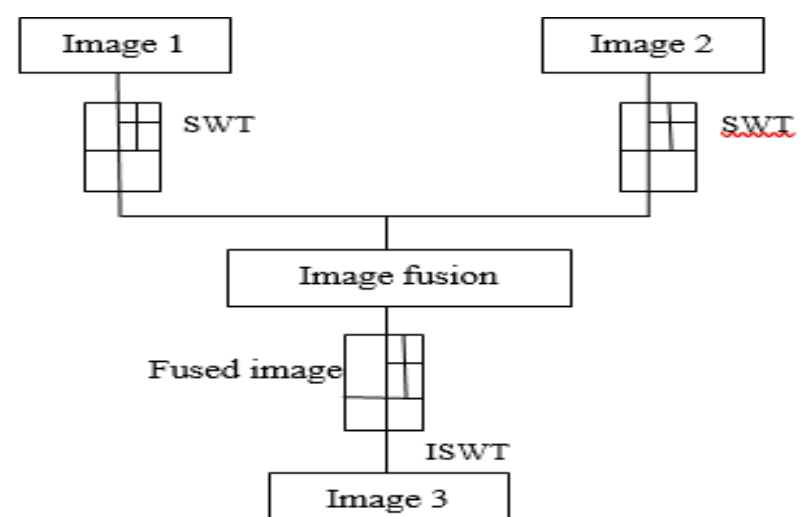

Fig 2: Fusion Techniques.

The aim of the image fusion concept is to display the fusion of two or more images that is combined into the new image that is enhanced in different ways and exhibits more detailed information. Various techniques were used to fuse the images, each having the advantages on their own way. The contrast of the image is measured and calculated by spatial frequencies to from each blocks of the source image if the blocks represent the largest frequencies to compose the image fusion.

Wavelet transform fusion method

The wavelet transform is used for the multi-focusing image focus that is mainly on high spatial frequency panchromatic image and the low spatial resolution image. Both image will combine and produce the highly spatial image that contains the information about the spectral image that is needed mostly in the medical domain. The result of the fused image contains the quality of multispectral imagery but it will distort the color information of the image in the process. As per the wavelet transform process the contrast of the images will be same because the fusion cannot take the contrast feature. The standard image fusion method perform rather more better than wavelet transform by reducing the color distortion when combining the wavelet method and standard scheme method it will produce the better result of the image fusion.

Contrast image fusion method

The contrast image fusion technique is based on the DCT domain It basically maintains the contrast color distortion. This method is used to measure and calculate the range of the contrast for some images which is analyzed and compared with other image fusion techniques to check if there is any difference between the fused image and wavelet based image and the visual quality. In the images the edges and outline of the object which is represented in the image is more important than the other information. To display the exact finicky area of the image by mixing the pixel replacement or contrast based image fusion method in wavelet transform domain.

\section{Proposed system}

Stationary Wavelet Transform: As we have seen the discrete wavelet transform (DWT) is not a time-invariant transform. The way to resolve the translation invariance is to some slightly different DWT, the so called stationary wavelet transforms (SWT). The stationary wavelet transform (SWT) is a wavelet transform algorithm designed to overcome the lack of translation invariance of discrete wavelet transform.

SWT algorithm does the suppressing of down-sampling and upsampling by inserting zeros between the sampling values. Translation-invariance is achieved by removing the down-samplers and up-samplers in the DWT. SWT removes redundant scheme in DWT and produces the output samples which contain the same number of input samples.

SWT will increase the contrast of the images, visual experiments and quantitative assessments demonstrate the effectiveness compared to present image fusion schemes, especially for medical diagnosis.

Input image:

An image that has been obtained as a result of the scan is stored electronically. Usually an image is described or represented in terms of vector graphics or raster graphics. The vector graphics creates the digital images through a sequence of commands or mathematical statements which place shapes and lines in a given plane whether two-dimensional or three-dimensional. This is the form of output that is usually obtained from the scans whether CT (Computed Tomography) or MRI (Magnetic Resonance Imaging). CT scan:

The Computerized Axial Tomography is actually the scan that combines the X-ray images together that is captured from different angles. The output of this scan is usually examined on a computer monitor. The area of examination is usually blood vessels, bones and soft tissues. Also it is the collection of various X-ray images that exclusively use computer software to view them. Since the Computed Tomography scan concentrated on the soft tissues and the Magnetic Resonance Imaging concentrates on the hard tissues, these scan outputs are given as input to the fusion process where the hard tissue details and the soft tissue details are combined to produce the high-resolution output.

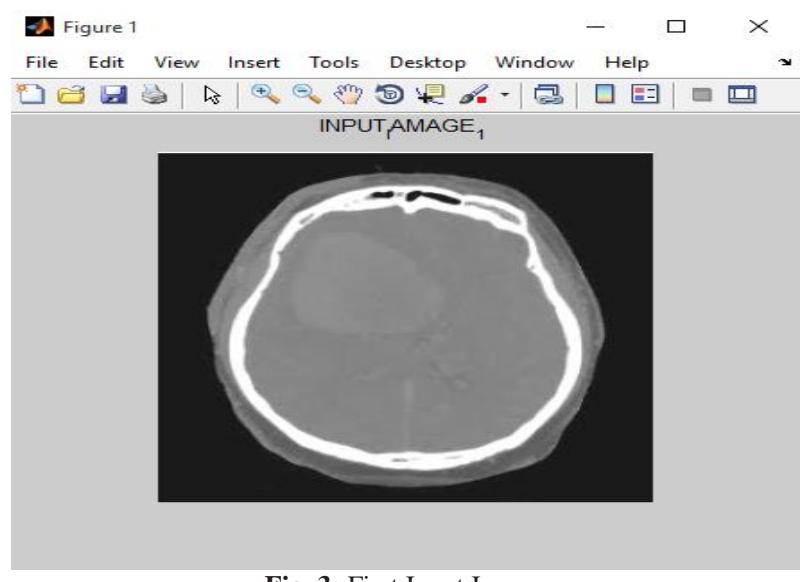

Fig. 3: First Input Image.

MRI Scan:

The detailed images of the body are obtained through this scan. The magnetic field and radio waves are used to produce the high detailed reproductions. These can penetrate and produce the images where even the X-rays can't see through. Also the added advantage of this scan is the high detail exhibiting nature of the output of the scan. It is highly used to examine the internal body structures and also with the help of this examination, anomalies such as tumors, strokes, aneurysms, etc. This is given as one of the inputs for the image fusion. 


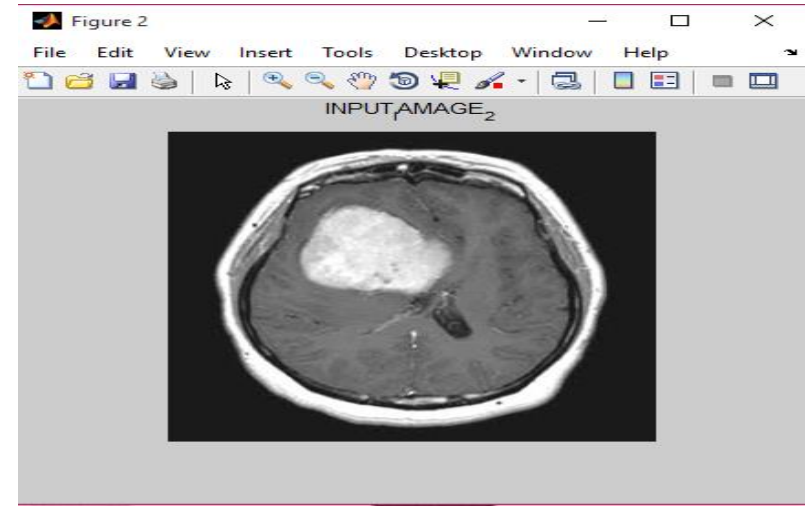

Fig. 4: Second Input Image.

Major Discussion:

The mass detection in brain MRI scan and Ishmic detection in brain CT scan and have compared the performance of this method with conventional method. The proposed algorithm offers the advantages of producing good quality segmentation and also easily visualizes the segmented region in three dimensional views for the treatment purpose. Histogram plot also measures based on the segmented output images from the input images.

Preprocessing Steps:

Before stepping into the actual fusing steps, it is a very vital and necessary to perform the action of preprocessing. A typical color image requires two matrices: A colormap and an Image matrix.

Colormap: Basically a colormap is a $3 \times 3$ matrix. It consists of real numbers as the elements. Each of the row in this matrix is a vector of RGB that defines a single color. It is also noted that they can be any length. The correspondence between the color and the data values that is provided depends on the type of visualization that is created. Each row of the colormap matrix is a $1 \times 3$ red, green, blue (RGB) color vector.

Color $=[R \mathrm{G} \mathrm{B}]$ that specifies the intensity of the red, green and blue components of that color. R, G and B are real scalars that range from 0.0 (black) to 1.0 (full intensity).

Image Matrix: For the fusion process to be implemented in the work-space, the images are represented as a two dimensional arrays. Here, each element of the matrix corresponds to a single pixel in the displayed image. To make the fusion process happen, it is a must process of conversion of the RGB plane to GRAY scale. Only after the conversion of the two images to a standard format that is the gray scale, the stationary wavelet transform wavelet can be applied on to them

In short, the colormap is an ordered set of values that represent the colors in the image. For each image pixel, the image matrix contains a corresponding index into the colormap.

The color components of an 8-bit RGB image are integers in the range $[0,255]$ rather than the floating point values in the range $[0$, 1]. The process involves the conversion of the RGB matrices into a gray-scale intensity image, using the standard perceptual weightings for the three-color components:

$\mathrm{Xrgb}=0.2990 \mathrm{R}+0.5870 \mathrm{G}+0.1140 \mathrm{~B}$

Then, the conversion of the gray-scale intensity image back to a gray-scale indexed image with 64 distinct levels is performed.

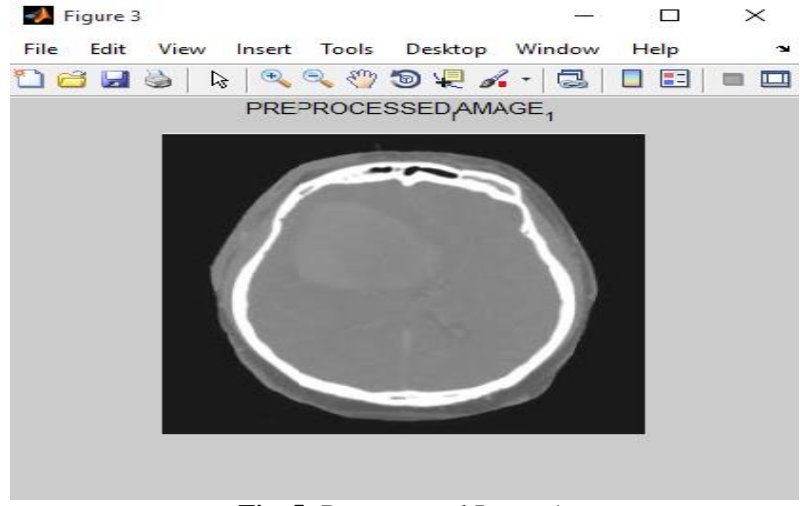

Fig. 5: Preprocessed Image 1.

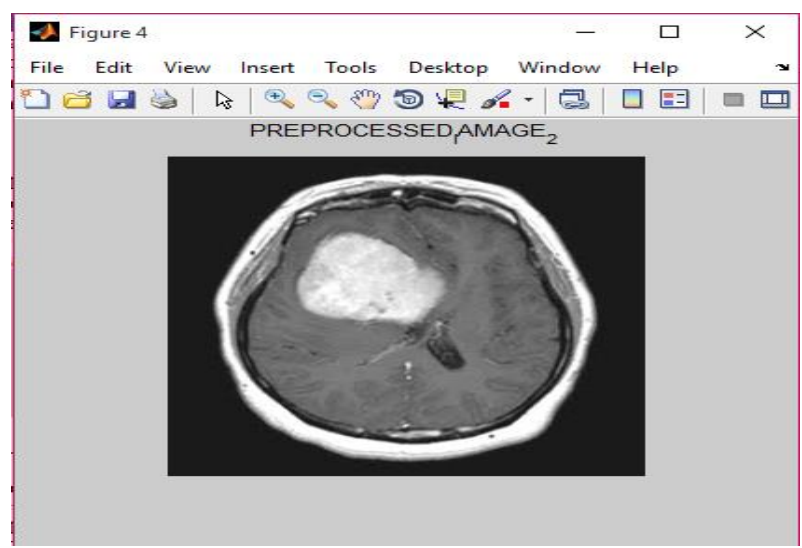

Fig. 6: Preprocessed Image 2.

Working of Algorithm

The SWT decompose the source image into rows and columns by low-pass and high-pass filtering and produce four sub-bands such as exact sub-band(LL) and edge detail sub-bands (LH, HL, HH).

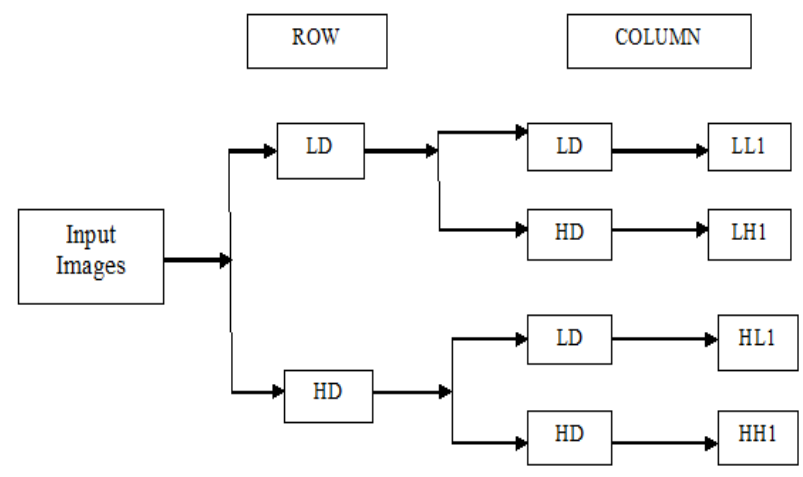

STRUCTURE OF SWT FILTER

Fig. 7: Structure of SWT Filter.

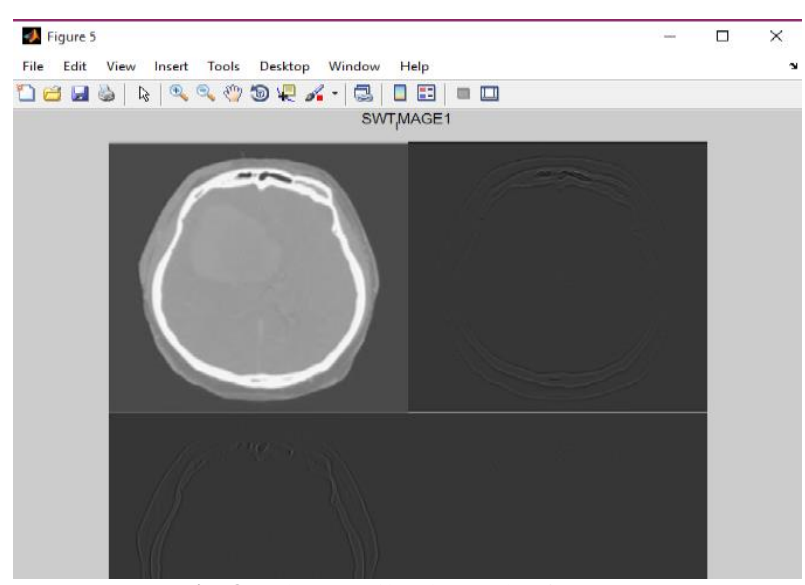

Fig. 8: Edge Detail Sub-Bands of Input1. 


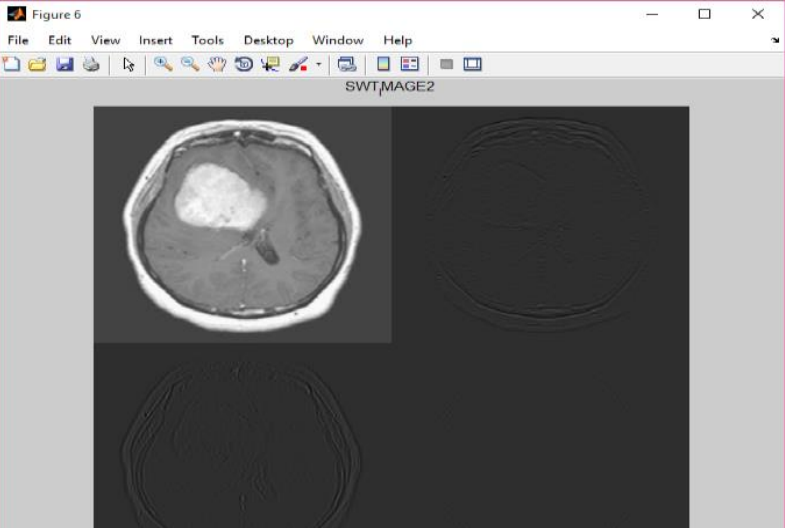

Fig. 9: Edge Detail Sub-Bands of Input2.

Performance of SWT

Two different source images are given as input. The two source images are divided into four bands as one exact sub-band and three edge details sub-bands (LL and LH, HL, HH). The LL band of two different images is combined to get the exact information about the images. The remaining LH, HL, HH bands of two source images are combined to get the edge information about source images. Once the images are combined, the contrast for the image is calculated. The contrast for the original image is calculated by applying the inverse stationary wavelet transform (ISWT). Main Architecture

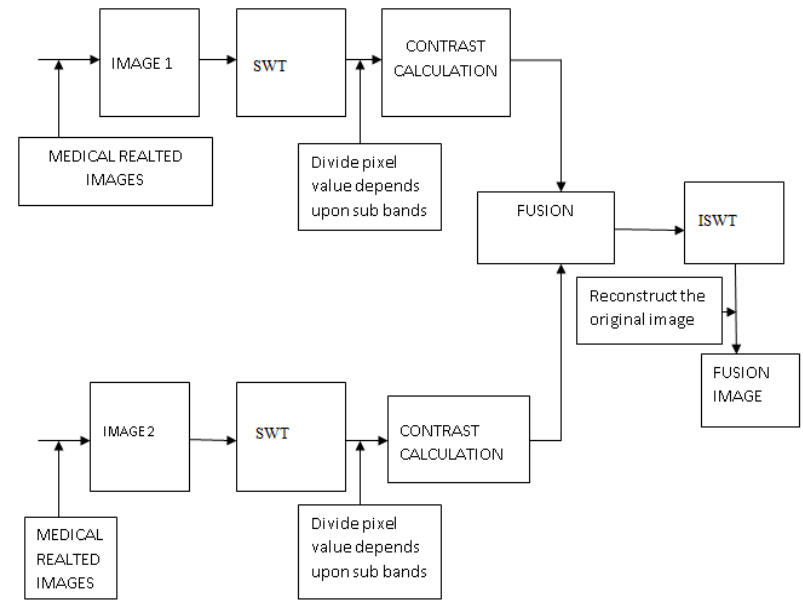

Fig. 10: Architecture Diagram.

\section{Resolution Enhancement}

To enhance the resolution, the noise is added in the low input resolution and low contrast image. The noise from the input image is removed using the median filter. The enhancement process is based on the interpolation of HF sub-band images obtained by SWT and the input image. The SWT comes into the scene to preserve the quality of high frequency components of the image. Also the reproduce the image in a more quality manner, it is very essential to preserve the edge information. Since the SWT has the equal length wavelet coefficients at each level along with redundant information, it makes the SWT shift-invariant and that makes features of edge-separation and de-noising enabled. The corrected HF sub-band and interpolated input are combined through ISWT, thus obtained the high resolution image. It is also observed the output has the high probability of sharper edges than the interpolated images obtained by the interpolation of the image directly.

Contrast Enhancement

The input to the module for enhancing the contrast is the resolution enhanced version of the image. The main tools used in the enhancement of contrast are singular value decomposition (SVD) and SWT. Even if the HF components are separated and some transformations are applied on the LF, will not cause any damage to the edge components. The key steps are: At first the SWT is applied on the input image and in contrast is improved using GHE.
Then the hanger (U), aligner (V) and singular value matrix (SVM) for the LL sub-bands are obtained. Then the above components are resolved as an equation and thus happen the improvement of the resolution.

Weiner Filtering:

There is a high tendency for the output of the scan images that is going to be the input of the fusion process, to be noisy and there is an inevitable need for the time invariant linear filtering to be applied on the image. And that is where the Weiner filtering comes into preprocessing step to remove the noises, blurs to be removed from the image before being displayed as the high detailed image as a result of the fusion technique. Thus it is an important prerequisite of the fusion process. The weiner filtering is optimal in terms of mean square error.

For this filter to be implemented, the power spectra of the original image and the additive noise are estimated. It is based statistical approach.

The estimated results are displayed in a cascaded implementation of inverse filtering and noise smoothing. It is a linear estimation of the original image.

Not only the deconvolution by inverse filtering (high pass filtering) is performed but also removes the noise with the compression operation (low pass filtering).

Highlights of Proposed System:

The potential advantages of the image fusion include: The reduction of the storage capacity by compiling the data apparent in two images into a single fused one. Image fusion not only avoids the redundant information produced by the sensor but also increases upon the diagnostic accuracy. Multimodal image fusion is broadly classified into three categories namely:

i) Pixel level

ii) Feature level and

iii) Decision level

Pixel level image fusion avoids the transformation of the pixel, thus preserving the original measured quantity while feature level highlights upon the edges, textures and fine details. Lastly, the decision level fusion deals with the selection of the features based on methods like fuzzy logic, voting and forecast.

\section{Conclusions}

In this paper, a new idea of fusing the CT scan and the MRI scan which are given as input images to the application developed, with a most effective and most recent image fusion algorithm Stationary Wavelet Transform is proposed. Also from the resulting fused image output, the status of the corresponding body organ is diagnosed automatically in the scenario of most common and also the rare cases, with the help of already stored multiple possibilities. The SWT image fusion technique is used in order to get the edge details in a more effective way which in turn helps in getting the more detailed view of the organ concerned. This is concentration on the edge information is more vital when it comes to the automated diagnosis of the disease. Also in the future, the upgradation to improve this idea to a more automated form where the application will be made more user friendly, where the diagnosis will be more accurate and the exact location of the affected cells will be taken in and also the proper medication will be displayed as output.

\section{References}

[1] S. Bauer et al., "A survey of MRI-based medical image analysis for brain tumour studies," Physics in medicine and biology, vol. 58, no. 13, pp. 97-129, 2013.

[2] D. N. Louis et al., "The 2007 who classification of tumoursof the central nervous system," Actaneuropathologica, vol. 114, no. 2, pp. 97-109, 2007

[3] E. G. Van Meir et al., "Exciting new advances in neurooncology: The avenue to a cure for malignant glioma," CA: a cancer journal for clinicians, vol. 60, no. 3, pp. 166-193, 2010. 
[4] G. Tabatabai et al., "Molecular diagnostics of gliomas: the clinical perspective," Acta neuropathologica, vol. 120, no. 5, pp. 585-592, 2010.

[5] B. Menze et al., "The multimodal brain tumour image segmentation benchmark (brats)," IEEE Transactions on Medical Imaging, vol. 34, no. 10, pp. 1993-2024, 2015.

[6] N. J. Tustison et al., "N4itk: improved n3 bias correction," IEEE Transactions on Medical Imaging, vol. 29, no. 6, pp. 1310-1320, 2010.

[7] L. G. Ny'ul, J. K. Udupa, and X. Zhang, "New variants of a method of MRI scale standardization," IEEE Transactions on Medical Imaging, vol. 19, no. 2, pp. 143-150, 2000. 